\title{
Double burden of underweight and overweight among Bangladeshi adults differs between men and women: evidence from a nationally representative survey
}

\author{
Mohammad E Hoque ${ }^{1, *}$, Md T Hasan ${ }^{1}$, Muntasirur Rahman ${ }^{1}$, Kurt Z Long ${ }^{1,2}$ and Abdullah \\ Al Mamun ${ }^{1}$ \\ ${ }^{1}$ School of Public Health, University of Queensland, Brisbane, QLD 4006, Australia: ${ }^{2}$ Department of Epidemiology \\ and Public Health, Swiss Tropical and Public Health Institute, Basel, Switzerland
}

Submitted 7 November 2015: Final revision received 4 March 2017: Accepted 13 April 2017: First published online 21 June 2017

\begin{abstract}
Objective: To estimate the prevalence of underweight and overweight among Bangladeshi adults and to determine if the double burden of underweight and overweight differs by gender and other socio-economic characteristics of individuals.

Design: We used data from the Bangladesh Demographic and Health Survey 2011. Multinominal logistic regression was used to examine associations between the different nutritional statuses of individuals and related determinants. Interaction effect was checked between gender and various socio-economic factors.

Setting: Nationwide, covering the whole of Bangladesh.

Subjects: Individuals aged >18 years (women, $n$ 16052; men, $n$ 5090).

Results: Underweight was observed among $28.3 \%$ of men and $24.4 \%$ of women, whereas overweight was observed among $8.4 \%$ of men and $16.9 \%$ of women. The odds of being overweight were significantly lower among urban men $(\mathrm{OR}=0.46 ; 95 \% \mathrm{CI} 0.37,0.57)$ compared with urban women, whereas the odds of being underweight were significantly higher among urban men $(\mathrm{OR}=1.33$; $95 \%$ CI $1.07,1.64)$ compared with urban women. The odds of being overweight were lower among higher educated men (OR $=0.48 ; 95 \% \mathrm{CI} 0.39,0.58)$ and men of rich households (OR $=0.45 ; 95 \%$ CI $0.37,0.54)$ compared with higher educated women and women of rich households, respectively.

Conclusions: There are important gender differences in the prevalence of underweight and overweight among the adult population in Bangladesh. Women with higher education, in rich and urban households have higher chances of being overweight and lower chances of being underweight compared with their male counterparts.
\end{abstract}

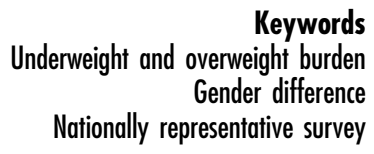

In the last several decades, many low- and middle-income countries including Bangladesh have experienced declines in the prevalence of underweight ${ }^{(1-3)}$ while facing an increase in the prevalence of overweight ${ }^{(1,3-6)}$. Both underweight and overweight are important determinants of disease-specific mortality and morbidity. Underweight is associated with co-morbidities such as osteoporosis, infertility and asthma ${ }^{(7,8)}$, while overweight and obesity are associated with CVD, cancer, non-insulin-dependent diabetes mellitus and hypertension ${ }^{(9,10)}$. In the case of Bangladesh, approximately $28.0 \%$ of rural women and $13.5 \%$ of urban women are classified as underweight ${ }^{(6)}$, while 11.4-28.9\% of women in urban areas and $1 \cdot 7-12 \cdot 1 \%$ of women in rural areas are overweight ${ }^{(5,6)}$. A recent study considering women of reproductive age showed that the prevalence of overweight has exceeded that of underweight in Bangla$\operatorname{desh}^{(11)}$. The increased burden of overweight ${ }^{(2,12)}$, along with the continued high prevalence of underweight, is of increasing concern for the overall health status of the country's population as well as for the health system ${ }^{(2)}$.

Globally few studies ${ }^{(13,14)}$ have focused on the gender differences in BMI and underweight and overweight prevalences at the population level. One study reported that in low- and middle-income countries women have higher BMI than men ${ }^{(13)}$. In addition, another study mentioned that more women in developed countries are overweight and obese compared with their male counterparts ${ }^{(14)}$. However, no such evidence for a gender difference in BMI in Bangladesh has been found in the literature, except for one study ${ }^{(15)}$ which found that women in rural areas had a 
greater prevalence of overweight than men. In addition, the Global Burden of Disease study ${ }^{(14)}$ reported that overweight and obesity are more prevalent among Bangladeshi women than men.

Several studies in Bangladesh have reported the prevalence of underweight and overweight among women of reproductive age and identified possible socioeconomic determinants ${ }^{(8,16-19)}$. However, such findings were not observed among men in Bangladesh, suggesting the need to examine whether different combinations of risk factors for underweight and overweight exist among men and women. It is important to determine gender differences in the double burden of underweight and overweight in Bangladesh and identify individual and household risk factors that contribute to the difference. Findings of such studies will have important public health implications to control and reduce the double burden of underweight and overweight in the country.

In 2011, the Bangladesh Demographic and Health Survey (BDHS) began to collect additional information on the nutritional status of both men and women across all ages $^{(6)}$. These data offer the opportunity to analyse gender differences in underweight and overweight status of individuals. Accordingly, the aim of the present study was to estimate the prevalence of underweight and overweight among adults in Bangladesh and to determine if the double burden of underweight and overweight differs by gender and other socio-economic characteristics of individuals. It was hypothesized that both underweight and overweight exist in men and women, and overweight prevalence is higher than underweight prevalence among both men and women.

\section{Methods}

\section{Data source}

Data for the present study came from BDHS 2011 which was conducted between July and December 2011. Demographic and Health Surveys are nationally representative, large-scale surveys that collect data on health, population and nutrition ${ }^{(6)}$. The BDHS 2011 used a two-stage stratified sampling design, where the first stage consisted of a sample of 600 enumeration areas or primary sampling units selected independently from the list of enumeration areas available from the 2011 housing and population census ${ }^{(20)}$. In the second stage, households were selected systematically from the chosen primary sampling units. All women of reproductive age (14-49 years) in the households were selected for interview and were asked to participate in the survey ${ }^{(6)}$. In a sub-sample of one-third of the households, all ever married men aged 15-54 years were selected and interviewed for the male survey. In addition, in households selected for the male survey, all men and women of the household aged 35 years or older were selected for a biomarker component that included height and weight along with blood pressure measurements, anaemia and blood glucose testing. The details of the sampling methodology, survey instrument and quality control procedures are reported elsewhere ${ }^{(6)}$. Informed consent was obtained from each participant before starting the interview.

\section{Study population and sample size}

According to the 2011 census, the total population of the country was 142 million and $49 \%$ of the total population were women ${ }^{(20)}$. About $34 \%$ of the total population lives in urban areas and $17 \%$ of the total population is aged 15 years or above ${ }^{(21)}$. About $74 \%$ of the population has completed primary education ${ }^{(21)}$. The data used in the present study were extracted from the individual data set of BDHS 2011 and consisted of 19466 females and 5254 males whose height, weight and other basic demographic and health data were available. Data were restricted to individuals who were residents of the sampled household, aged greater than 18 years old and women who were not pregnant at the time of the survey. The final sample comprised 16052 women and 5090 men from 17141 residential households.

\section{Measurement of $\mathrm{BMI}$}

In BDHS 2011, weight and height of the individuals were collected using standardized procedures. Weight and height were measured by trained personnel using a solarpowered scale with accuracy of $0.1 \mathrm{~kg}$ and a standardized measuring board with accuracy of $0 \cdot 1 \mathrm{~cm}$, respectively ${ }^{(6)}$. BMI, calculated as weight in kilograms divided by the square of height in metres $\left(\mathrm{kg} / \mathrm{m}^{2}\right)$, was the outcome for the present study. Following the WHO expert consultation meeting ${ }^{(22,23)}$, the following cut-offs were used: underweight $\left(\mathrm{BMI}<18.5 \mathrm{~kg} / \mathrm{m}^{2}\right)$, normal BMI $\quad(\mathrm{BMI}=18.5-$ $24.9 \mathrm{~kg} / \mathrm{m}^{2}$ ) and overweight (BMI $\left.\geq 25.0 \mathrm{~kg} / \mathrm{m}^{2}\right)$.

\section{Measurement of potential determinants}

The current study used a comprehensive list of variables representing socio-economic and demographic dimensions as potential determinants which may affect nutritional status of the adult population in Bangladesh based on the available information in the data set. Age and gender of the respondent, educational attainment, region, wealth tertile, urban living, current marital status and current working status were considered as potential determinants for the BMI of adults. The age of the respondent was grouped into three categories: 19-34, $35-49$ and $\geq 50$ years. The lower bound of 19 years of age was used since individuals are considered adolescents until 18 years of age ${ }^{(20)}$. The education of the respondent was grouped into three categories: no education, primary education, and secondary or higher education. The wealth tertile is an indicator of relative wealth according to asset holdings, which was constructed using factor analysis ${ }^{(24)}$. We re-categorized wealth index into three groups: poor, 
middle and rich. A variable indicating whether the respondent was living in an urban or rural area the time of the interview was included in the analysis. Variables indicating the marital status of the respondent and currently working or not working were also included in the analysis.

\section{Statistical analysis}

The prevalence of underweight and overweight by gender was first calculated and compared. Multinominal logistic regression was then used to examine associations between the different nutritional categories (underweight, normal BMI and overweight) and the specific socioeconomic and demographic factors. In these analyses both adjusted and unadjusted odds ratios were calculated, separately for men and women. The adjusted models incorporated age of the respondents, residence, region, wealth tertile, educational attainment, marital status and working status as potential confounders. Statistical significance was set at a probability level of $<0 \cdot 05$. Interaction terms between gender and socio-economic and demographic characteristics were also included in the model. The LINCOM procedure available in the STATA statistical software package was used to calculate the interaction effect. All analyses were carried out using STATA version 12 and included sampling weights to account for non-proportional allocation of the sample based on administrative divisions and urban/rural areas in Bangladesh. Variables that were found to have a $P$ value $<0.05$ in the multinominal analysis were then included in multiple linear regression analysis to predict their effect on mean BMI for men and women.

\section{Results}

The mean age for men and women was $45 \cdot 5$ (SD 15.3) and $35 \cdot 5$ (SD 12.2) years, respectively. Three-quarters of the individuals, irrespective of gender, were from rural areas. Men were more likely to have a higher education (36.7 $v$. $35.3 \% ; P=0.0474)$ and be employed at the time of the survey $(89.4 v .14 .3 \% ; P<0.001)$ compared with women. The majority of men $(97 \cdot 6 \%)$ and women $(89.2 \%)$ were currently married $(P<0 \cdot 001)$.

Table 1 shows the prevalence of underweight and overweight among men and women by different socioeconomic characteristics. The overweight prevalence in women was double that in men (16.9 v. 8.4\%, $P<0.001)$. The prevalence of overweight was higher among women compared with men across all age groups, wealth tertiles and levels of education. Underweight prevalence was $28.3 \%$ in men and $24.4 \%$ in women $(P<0.001)$, whereas $8.4 \%$ of men and $16.9 \%$ of women were overweight. The prevalence of underweight in rural areas was 31.4 and $28.1 \%$, and in urban areas was 18.7 and $13.4 \%$, among males and females, respectively. On the other hand, the prevalence of overweight in rural areas was 5.6 and $12.5 \%$, and in urban areas was 17.1 and $29.9 \%$, among males and females, respectively. Overweight prevalence increased gradually with wealth status of the household

Table 1 Nutritional status of men and women aged 19 years or above by background characteristics, Bangladesh Demographic and Health Survey 2011

\begin{tabular}{|c|c|c|c|c|c|c|c|c|c|c|}
\hline & \multicolumn{5}{|c|}{ Males } & \multicolumn{5}{|c|}{ Females } \\
\hline & $n$ & $\%$ & $\begin{array}{c}\text { Normal BMI } \\
(\%)\end{array}$ & $\begin{array}{c}\text { Underweight } \\
(\%)\end{array}$ & $\begin{array}{c}\text { Overweight } \\
(\%)\end{array}$ & $n$ & $\%$ & $\begin{array}{c}\text { Normal BMI } \\
(\%)\end{array}$ & $\begin{array}{c}\text { Underweight } \\
(\%)\end{array}$ & $\begin{array}{c}\text { Overweight } \\
(\%)\end{array}$ \\
\hline \multicolumn{11}{|l|}{ Age category } \\
\hline 19-34 years & 1368 & $26 \cdot 8$ & $67 \cdot 1$ & $26 \cdot 3$ & $6 \cdot 6$ & 8506 & 53.0 & $61 \cdot 2$ & 23.5 & $15 \cdot 3$ \\
\hline $35-49$ years & 1817 & $35 \cdot 7$ & $66 \cdot 1$ & 22.5 & 11.4 & 5989 & 37.3 & 57.6 & $22 \cdot 4$ & $20 \cdot 0$ \\
\hline$\geq 50$ years & 1905 & 37.5 & $57 \cdot 9$ & $35 \cdot 2$ & 6.9 & 1556 & $9 \cdot 7$ & $49 \cdot 6$ & $36 \cdot 5$ & 13.9 \\
\hline \multicolumn{11}{|l|}{ Residence } \\
\hline Rural & 3827 & $75 \cdot 2$ & 63.0 & 31.4 & $5 \cdot 6$ & 12015 & 74.9 & $59 \cdot 4$ & $28 \cdot 1$ & $12 \cdot 5$ \\
\hline Urban & 1263 & 24.8 & $64 \cdot 1$ & $18 \cdot 7$ & $17 \cdot 1$ & 4036 & $25 \cdot 1$ & $56 \cdot 7$ & 13.4 & 29.9 \\
\hline \multicolumn{11}{|l|}{ Wealth tertile } \\
\hline Poor & 2028 & $39 \cdot 8$ & $60 \cdot 2$ & 38.2 & 1.6 & 6186 & 38.5 & 58.4 & 35.4 & $6 \cdot 2$ \\
\hline Middle & 992 & 19.5 & $64 \cdot 3$ & 30.5 & $5 \cdot 2$ & 3192 & $20 \cdot 0$ & $62 \cdot 6$ & $25 \cdot 7$ & 11.7 \\
\hline Rich & 2070 & $40 \cdot 7$ & 65.9 & 17.5 & $16 \cdot 6$ & 6673 & 41.5 & $57 \cdot 2$ & 13.5 & $29 \cdot 3$ \\
\hline \multicolumn{11}{|c|}{ Highest education level } \\
\hline No education & 1697 & 33.3 & $58 \cdot 1$ & $39 \cdot 4$ & 2.5 & 5660 & $35 \cdot 3$ & $57 \cdot 6$ & 31.9 & 10.5 \\
\hline Primary & 1524 & $30 \cdot 0$ & 65.4 & $28 \cdot 2$ & 6.4 & 4717 & $29 \cdot 4$ & 59.9 & $25 \cdot 0$ & $15 \cdot 1$ \\
\hline $\begin{array}{l}\text { Secondary or } \\
\text { higher }\end{array}$ & 1869 & $36 \cdot 7$ & $66 \cdot 3$ & $18 \cdot 3$ & $15 \cdot 4$ & 5674 & $35 \cdot 3$ & $58 \cdot 9$ & $16 \cdot 4$ & $24 \cdot 7$ \\
\hline \multicolumn{11}{|l|}{ Current marital status } \\
\hline Not married & 121 & $2 \cdot 3$ & 49.7 & 44.9 & 5.4 & 1738 & $10 \cdot 8$ & $50 \cdot 1$ & $36 \cdot 1$ & 13.8 \\
\hline Currently married & 4969 & $97 \cdot 6$ & 63.6 & $27 \cdot 9$ & 8.5 & 14314 & $89 \cdot 2$ & $59 \cdot 8$ & $22 \cdot 9$ & $17 \cdot 3$ \\
\hline \multicolumn{11}{|l|}{ Current working status } \\
\hline Currently working & 4553 & 89.4 & 64.5 & $26 \cdot 9$ & 8.6 & 2287 & $14 \cdot 3$ & $59 \cdot 3$ & 23.6 & $17 \cdot 1$ \\
\hline Not working & 537 & $10 \cdot 6$ & 53.1 & 39.9 & $7 \cdot 0$ & 13765 & $85 \cdot 7$ & 58.6 & 24.5 & $16 \cdot 9$ \\
\hline
\end{tabular}


and education level for both genders. Higher prevalence of underweight was observed among both males and females who were not currently married and the opposite was true for overweight prevalence for both genders. On the other hand, underweight was high for both males and females who are not working and the overweight prevalence was lower among both genders for the same characteristic.

Table 2 demonstrates the unadjusted and adjusted odds ratios for the associations between underweight and overweight and different sociodemographic characteristics of the respondents. Living in an urban area, belonging to higher wealth tertiles and being more educated were all inversely associated with underweight and directly associated with overweight among both men and women. The odds of being overweight were significantly greater among men and women in the highest wealth tertile compared with men and women in the lowest, respectively. In contrast, the odds of being underweight were significantly lower among men and women in the rich tertile compared with men and women in the poor tertile, respectively. The odds of being overweight were significantly higher among men and women with a secondary or higher education level compared with non-educated men and women. In addition, men in the same higher education category were $43 \%$ less likely to be underweight compared with non-educated men, whereas secondary or higher educated women were $23 \%$ less likely to be underweight compared with non-educated women.

The interaction model results presented in Table 3 show that the odds of being overweight were significantly lower among men living in rural areas (OR $=0.37 ; 95 \% \mathrm{CI} 0.30$, $0.46)$ compared with women living in rural areas. The odds of being overweight were lower among secondary or higher educated men compared with secondary or higher educated women $(\mathrm{OR}=0.48 ; 95 \%$ CI 0.39, 0.58); however, no significant difference was observed in odds of being underweight between the secondary or higher educated men and women $(\mathrm{OR}=1.04 ; 95 \% \mathrm{CI} 0.85,1.28)$. The odds of overweight were also lower among men from highertertile households compared with women from such

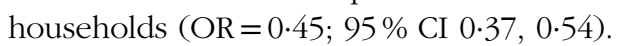

Table 4 presents the unadjusted and adjusted mean BMI for men and women. The effect of different determinants on mean BMI varied substantially. Mean BMI was higher among urban residents compared with rural residents. Mean BMI was also higher among individuals who were currently married, from a higher wealth tertile and more educated. The effect of different determinants on mean BMI was different for men and women, as shown in Table 5. Mean BMI among men aged 35-49 years was $-1.08(95 \%$ CI $-1.31,-0.86) \mathrm{kg} / \mathrm{m}^{2}$ lower than among women aged $35-49$ years. Similarly, men aged $\geq 50$ years had a BMI on average $-1.06(95 \% \mathrm{CI}-1.35,-0.77) \mathrm{kg} / \mathrm{m}^{2}$ lower compared with women of the same age. Mean BMI was $-1.47(95 \% \mathrm{CI}-1.73,-1.21) \mathrm{kg} / \mathrm{m}^{2}$ lower among men living in urban areas compared with urban women. Men who had secondary or higher education had a BMI on average $-0.94(95 \% \mathrm{CI}-1.18,-0.69) \mathrm{kg} / \mathrm{m}^{2}$ lower compared with their female counterparts who had similar level of education. Men from higher wealth tertiles had significantly lower mean BMI compared with women from the same tertiles.

\section{Discussion}

To the best of our knowledge, the present study the first to examine several important modifiable and non-modifiable determinants associated with underweight and overweight among Bangladeshi men and women, based on nationally representative data. As such the study provides information on the patterns of underweight and overweight among adult male and female populations in Bangladesh who are undergoing a nutritional transition ${ }^{(1,2)}$ from underweight burden to a double burden of underweight and overweight. Factors associated with underweight and overweight status of women have been studied in Bangladesh $^{(11,17,18)}$, but no such study is available for Bangladeshi men. Our findings demonstrate the clear existence of a double burden of underweight and overweight among both genders, although underweight dominates in both cases, except among women from urban areas. Among the latter, the overweight prevalence (29.9\%) is more than double the underweight prevalence (13.4\%). About $28 \%$ of men and $25 \%$ of women are underweight, whereas $8 \%$ of men and $17 \%$ of women are overweight.

Our findings are similar to other studies on men and women in Bangladesh ${ }^{(6,25)}$. They are also in agreement with the higher prevalence of overweight among women reported from the Global Burden of Disease studies ${ }^{(14)}$. Women had significantly higher mean BMI than men $(21.5 v$. $20.6 \mathrm{~kg} / \mathrm{m}^{2}$ ), which is consistent with the findings of studies carried out in other parts of $\mathrm{Asia}^{(9,19,24)}$. It is also consistent with findings globally that women have a higher mean BMI than men, although it was reported that men had higher BMI than women in high-income regions of the world ${ }^{(9)}$.

The present study reveals that women with high wealth status, higher education attainment and urban residence had higher risk of being overweight and lower risk of being underweight, compared with their male counterparts. Few previous studies ${ }^{(9)}$ have reported that women have a higher prevalence of overweight compared with men. However, the present study adds new knowledge to the literature by showing that the risk factors that are associated with underweight and overweight differ by gender and other socio-economic characteristics of individuals. In the model incorporating interaction terms, urban residence, secondary or higher education and higher wealth status were consistently protective against overweight among men compared with women from rural areas, uneducated women and poor women. Additionally, 
Table 2 Adjusted and unadjusted odds ratios for the associations between underweight and overweight among men and women and different characteristics, Bangladesh Demographic and Health Survey 2011

\begin{tabular}{|c|c|c|c|c|c|c|c|c|c|c|c|c|c|c|c|c|c|c|c|c|}
\hline & \multicolumn{10}{|c|}{ Males } & \multicolumn{10}{|c|}{ Females } \\
\hline & \multicolumn{5}{|c|}{ Unadjusted } & \multicolumn{5}{|c|}{ Adjusted } & \multicolumn{5}{|c|}{ Unadjusted } & \multicolumn{5}{|c|}{ Adjusted } \\
\hline & \multirow{2}{*}{$\begin{array}{c}\begin{array}{c}\text { Normal } \\
\text { BMI }\end{array} \\
\text { OR }\end{array}$} & \multicolumn{2}{|c|}{ Underweight } & \multicolumn{2}{|c|}{ Overweight } & \multirow{2}{*}{$\begin{array}{c}\begin{array}{c}\text { Normal } \\
\text { BMI }\end{array} \\
\text { OR }\end{array}$} & \multicolumn{2}{|c|}{ Underweight } & \multicolumn{2}{|c|}{ Overweight } & \multirow{2}{*}{$\begin{array}{c}\begin{array}{c}\text { Normal } \\
\text { BMI }\end{array} \\
\mathrm{OR}\end{array}$} & \multicolumn{2}{|c|}{ Underweight } & \multicolumn{2}{|c|}{ Overweight } & \multirow{2}{*}{$\begin{array}{c}\begin{array}{c}\text { Normal } \\
\text { BMI }\end{array} \\
\text { OR }\end{array}$} & \multicolumn{2}{|c|}{ Underweight } & \multicolumn{2}{|c|}{ Overweight } \\
\hline & & OR & $95 \% \mathrm{Cl}$ & OR & $95 \% \mathrm{Cl}$ & & OR & $95 \% \mathrm{Cl}$ & OR & $95 \% \mathrm{Cl}$ & & OR & $95 \% \mathrm{Cl}$ & OR & $95 \% \mathrm{Cl}$ & & OR & $95 \% \mathrm{Cl}$ & OR & $95 \% \mathrm{Cl}$ \\
\hline & & & & & & & & & & & & & & & & & & & & \\
\hline $\begin{array}{l}19-34 \text { years } \\
35-49 \text { years }\end{array}$ & $\begin{array}{l}1.00 \\
1.00\end{array}$ & $\begin{array}{l}1.00 \\
0.86\end{array}$ & $\begin{array}{c}\text { Ref. } \\
0.72,1.04\end{array}$ & $\begin{array}{l}1.00 \\
1.77^{* \star *}\end{array}$ & $\begin{array}{c}\text { Ref. } \\
1 \cdot 36,2 \cdot 33\end{array}$ & $\begin{array}{l}1.00 \\
1.00\end{array}$ & $\begin{array}{l}1.00 \\
0.81^{\star}\end{array}$ & $\begin{array}{c}\text { Ref. } \\
0.67,0.97\end{array}$ & $\begin{array}{l}1.00 \\
1.92^{\star \star \star}\end{array}$ & $\begin{array}{c}\text { Ref. } \\
1.45,2 \cdot 55\end{array}$ & $\begin{array}{l}1.00 \\
1.00\end{array}$ & $\begin{array}{l}1.00 \\
1.01\end{array}$ & $\begin{array}{l}\text { Ref. } \\
0.92,1 \cdot 11\end{array}$ & $\begin{array}{l}1.00 \\
1.39^{* \star \star}\end{array}$ & $\begin{array}{c}\text { Ref. } \\
1.25,1.54\end{array}$ & $\begin{array}{l}1.00 \\
1.00\end{array}$ & $\begin{array}{l}1.00 \\
0.89^{*}\end{array}$ & $\begin{array}{c}\text { Ref. } \\
0.80,0.98\end{array}$ & $\begin{array}{l}1.00 \\
1.65^{\star \star \star}\end{array}$ & $\begin{array}{c}\text { Ref. } \\
1.48,1.84\end{array}$ \\
\hline $\begin{array}{l}\geq 50 \text { years } \\
\text { esidence }\end{array}$ & 1.00 & $1.55^{* \star \star}$ & $1.31,1.84$ & 1.23 & $0.91,1.65$ & 1.00 & $1 \cdot 27^{\star *}$ & $1.05,1.52$ & $1.51^{\star}$ & $1 \cdot 08,2.11$ & 1.00 & $1.91^{\star \star \star}$ & * $1.67,2 \cdot 19$ & 1.12 & $0.92,1.36$ & 1.00 & $1.39^{\star \star \star}$ & $1.18,1.65$ & $1.53^{\star \star}$ & $1.21,1.93$ \\
\hline $\begin{array}{l}\text { Rural } \\
\text { Urban }\end{array}$ & $\begin{array}{l}1.00 \\
1.00\end{array}$ & $\begin{array}{l}1.00 \\
0.58^{* * *}\end{array}$ & $\begin{array}{c}\text { Ref. } \\
0.47,0.71\end{array}$ & $\begin{array}{l}1.00 \\
3.03^{\star \star *}\end{array}$ & $\begin{array}{c}\text { Ref. } \\
2.37,3.87\end{array}$ & $\begin{array}{l}1.00 \\
1.00\end{array}$ & $\begin{array}{l}1.00 \\
0.88\end{array}$ & $\begin{array}{c}\text { Ref. } \\
0.71,1.08\end{array}$ & $\begin{array}{l}1.00 \\
1.95^{\star \star \star}\end{array}$ & $\begin{array}{c}\text { Ref. } \\
1 \cdot 51,2 \cdot 52\end{array}$ & $\begin{array}{l}1.00 \\
1.00\end{array}$ & $\begin{array}{l}1.00 \\
0.50^{\star \star \star}\end{array}$ & $\begin{array}{c}\text { Ref. } \\
0.43,0.57\end{array}$ & $\begin{array}{l}1 \cdot 00 \\
2 \cdot 49^{* *}\end{array}$ & $\begin{array}{c}\text { Ref. } \\
2 \cdot 16,2 \cdot 88\end{array}$ & $\begin{array}{l}1.00 \\
1.00\end{array}$ & $\begin{array}{l}1.00 \\
0.72^{\star * \star}\end{array}$ & $\begin{array}{c}\text { Ref. } \\
0.63,0.83\end{array}$ & $\begin{array}{l}1.00 \\
1.54^{\star \star \star}\end{array}$ & $\begin{array}{c}\text { Ref. } \\
1.34,1.79\end{array}$ \\
\hline Wealth tertile & & & & & & & & & & & & & & & & & & & & \\
\hline $\begin{array}{l}\text { Poor } \\
\text { Middle } \\
\text { Rich }\end{array}$ & $\begin{array}{l}1.00 \\
1.00\end{array}$ & $\begin{array}{l}0.74^{\star \star} \\
0.4^{\star \star \star *}\end{array}$ & $\begin{array}{l}0.62,0.89 \\
0.35,0.49\end{array}$ & $\begin{array}{l}3.08^{\star \star \star} \\
9.56^{* \star *}\end{array}$ & $\begin{array}{l}1.85,5.13 \\
6.24,14 \cdot 63\end{array}$ & $\begin{array}{l}1.00 \\
1.00\end{array}$ & $\begin{array}{l}0.81^{\star} \\
0.53^{\star * \star}\end{array}$ & $\begin{array}{l}0.66,0.97 \\
0.43 \\
0.65\end{array}$ & $\begin{array}{l}2.46^{* *} \\
5.23^{* * *}\end{array}$ & $\begin{aligned} \text { rel. } \\
1.45,4.15 \\
3.27 .833\end{aligned}$ & $\begin{array}{l}1.00 \\
1.00\end{array}$ & $\begin{array}{l}0.67^{\star \star \star} \\
0.39^{\star \star \star}\end{array}$ & $\begin{array}{l}0.59,0.76 \\
\times \quad 0.35 \\
\times\end{array}$ & $\begin{array}{l}1.78^{* \star \star} \\
4.80^{* \star *}\end{array}$ & $\begin{array}{l}1.45,2 \cdot 10 \\
4.15 \\
4.54\end{array}$ & $\begin{array}{l}1.00 \\
1.00\end{array}$ & $\begin{array}{l}0.72^{\star \star \star} \\
0.47^{\star \star \star}\end{array}$ & $\begin{array}{l}0.63,0.82 \\
0.41 \\
0.54\end{array}$ & $\begin{array}{l}1.51^{\star \star \star} \\
3.26^{\star * \star}\end{array}$ & $\begin{array}{l}1.24,1.82 \\
2.81 .3 .78\end{array}$ \\
\hline Highest & & & & & & & & & & & & & & & & & & & & \\
\hline $\begin{array}{l}\text { No education } \\
\text { Primary }\end{array}$ & $\begin{array}{l}1.00 \\
1.00\end{array}$ & $\begin{array}{l}1.00 \\
0.63^{* \star *}\end{array}$ & $\begin{array}{l}\text { Ref. } \\
0.53,0.76\end{array}$ & $\begin{array}{l}1.00 \\
2.31^{* \star *}\end{array}$ & $\begin{array}{c}\text { Ref. } \\
1.47,3.64\end{array}$ & $\begin{array}{l}1.00 \\
1.00\end{array}$ & $\begin{array}{l}1.00 \\
0.72^{\star *}\end{array}$ & $\begin{array}{c}\text { Ref. } \\
0.59,0.87\end{array}$ & $\begin{array}{l}1.00 \\
1.78^{\star}\end{array}$ & $\begin{array}{l}\text { Ref. } \\
1 \cdot 13,2 \cdot 82\end{array}$ & $\begin{array}{l}1.00 \\
1.00\end{array}$ & $\begin{array}{l}1.00 \\
0.75^{\star * \star}\end{array}$ & $\begin{array}{c}\text { Ref. } \\
0.67,0.84\end{array}$ & $\begin{array}{l}1.00 \\
1.38^{\star \star \star}\end{array}$ & $\begin{array}{l}\text { Ref. } \\
1 \cdot 20,1 \cdot 60\end{array}$ & $\begin{array}{l}1.00 \\
1.00\end{array}$ & $\begin{array}{l}1.00 \\
0.89^{*}\end{array}$ & $\begin{array}{c}\text { Ref. } \\
0.79,1.01\end{array}$ & $\begin{array}{l}1.00 \\
1.31^{* *}\end{array}$ & $\begin{array}{l}\text { Ref. } \\
1.12,1.54\end{array}$ \\
\hline $\begin{array}{l}\text { Secondary or higher } \\
\text { Current marital status }\end{array}$ & 1.00 & $0.41^{\star \star \star}$ & $0.33,0.49$ & $5 \cdot 47^{\star \star \star}$ & $3 \cdot 69,8.11$ & 1.00 & $0.57^{\star \star \star}$ & $0.45,0.72$ & $2 \cdot 86^{* \star}$ & $1 \cdot 87,4.37$ & 1.0 & $0.50^{\star \star \star}$ & $0.44,0.56$ & $2 \cdot 30^{* * *}$ & $2 \cdot 02,2.62$ & 1.00 & $0.77^{\star \star}$ & $0.67,0.88$ & $1.73^{* \star *}$ & $1.50,2.00$ \\
\hline $\begin{array}{l}\text { Not married } \\
\text { Currently married } \\
\text { Current working st? }\end{array}$ & $\begin{array}{l}1.00 \\
1.00\end{array}$ & $\begin{array}{l}1.00 \\
0.48^{* *}\end{array}$ & $\begin{array}{c}\text { Ref. } \\
0.31,0.73\end{array}$ & $\begin{array}{l}1.00 \\
1.22\end{array}$ & $\begin{array}{c}\text { Ref. } \\
0.49,2 \cdot 99\end{array}$ & $\begin{array}{l}1.00 \\
1.00\end{array}$ & $\begin{array}{l}1.00 \\
0.58^{*}\end{array}$ & $\begin{array}{c}\text { Ref. } \\
0.38,0.89\end{array}$ & $\begin{array}{l}1.00 \\
1.24\end{array}$ & $\begin{array}{l}\text { Ref. } \\
0.49,3 \cdot 14\end{array}$ & $\begin{array}{l}1.00 \\
1.00\end{array}$ & $\begin{array}{l}1.00 \\
0.53^{* \star \star}\end{array}$ & $\begin{array}{c}\text { Ref. } \\
0.47,0.60\end{array}$ & $\begin{array}{l}1.00 \\
1.05\end{array}$ & $\begin{array}{c}\text { Ref. } \\
0.88,1.24\end{array}$ & $\begin{array}{l}1.00 \\
1.00\end{array}$ & $\begin{array}{l}1.00 \\
0.65^{* * *}\end{array}$ & $\begin{array}{c}\text { Ref. } \\
0.56,0.74\end{array}$ & $\begin{array}{l}1.00 \\
1.01\end{array}$ & $\begin{array}{l}\text { Ref. } \\
0.84,1.22\end{array}$ \\
\hline $\begin{array}{l}\text { Currently working } \\
\text { Not working }\end{array}$ & $\begin{array}{l}1.00 \\
1.00\end{array}$ & $\begin{array}{l}1.00 \\
0.55^{\star \star \star}\end{array}$ & $\begin{array}{c}\text { Ref. } \\
0.45,0.68\end{array}$ & $\begin{array}{l}1.00 \\
1.01\end{array}$ & $\begin{array}{c}\text { Ref. } \\
0.70,1.45\end{array}$ & $\begin{array}{l}1.00 \\
1.00\end{array}$ & $\begin{array}{l}1.00 \\
0.72\end{array}$ & $\begin{array}{c}\text { Ref. } \\
0.55,0.88\end{array}$ & $\begin{array}{l}1.00 \\
1.03\end{array}$ & $\begin{array}{c}\text { Ref. } \\
0.67,1.58\end{array}$ & $\begin{array}{l}1.00 \\
1.00\end{array}$ & $\begin{array}{l}1.00 \\
0.95\end{array}$ & $\begin{array}{c}\text { Ref. } \\
0.83,1.09\end{array}$ & $\begin{array}{l}1.00 \\
0.99\end{array}$ & $\begin{array}{c}\text { Ref. } \\
0.86,1.15\end{array}$ & $\begin{array}{l}1.00 \\
1.00\end{array}$ & $\begin{array}{l}1.00 \\
0.98\end{array}$ & $\begin{array}{c}\text { Ref. } \\
0.86,1.12\end{array}$ & $\begin{array}{l}1.00 \\
0.92\end{array}$ & $\begin{array}{c}\text { Ref. } \\
0.78,1.07\end{array}$ \\
\hline
\end{tabular}

Ref., reference category

${ }^{\star} P<0.05,{ }^{\star \star} P<0.01,{ }^{\star \star \star} P<0.001$. 
Table 3 Adjusted odds ratios in interaction models for underweight and overweight among men and women, Bangladesh Demographic and Health Survey 2011

\begin{tabular}{|c|c|c|c|c|c|c|}
\hline & \multicolumn{4}{|c|}{ Adjusted } & \multicolumn{2}{|c|}{ Overall test of interaction } \\
\hline & \multicolumn{2}{|c|}{ Underweight } & \multicolumn{2}{|c|}{ Overweight } & \multirow{2}{*}{$\frac{\text { Underweight }}{P \text { value }}$} & \multirow{2}{*}{$\frac{\text { Overweight }}{P \text { value }}$} \\
\hline & OR & $95 \% \mathrm{Cl}$ & OR & $95 \% \mathrm{Cl}$ & & \\
\hline $\begin{array}{l}\text { Model 1: age } \times \text { gender } \\
\text { Gender (ref.: women) }\end{array}$ & & & & & 0.7048 & 0.3024 \\
\hline Men & $1 \cdot 15$ & $0.95,1.37$ & $0.40^{\star \star \star}$ & $0.30,0.53$ & & \\
\hline $\begin{array}{l}\text { Age category (ref.: } 19-34 \text { years) } \\
35-49 \text { years } \\
\geq 50 \text { years } \\
\text { (Men } v \text {. women }) \times 35-49 \text { years } \\
\text { (Men } v \text {. women) } \times \geq 50 \text { years }\end{array}$ & $\begin{array}{l}0 \cdot 86^{\star \star *} \\
1 \cdot 32^{\star \star *} \\
1 \cdot 10 \\
1.21\end{array}$ & $\begin{array}{l}0.78,0.95 \\
1.12,1.55 \\
0.92,1.31 \\
0.99,1.47\end{array}$ & $\begin{array}{l}1.69^{\star \star \star} \\
1.59^{\star \star \star} \\
0.44^{\star \star \star} \\
0.35^{\star \star \star}\end{array}$ & $\begin{array}{l}1.51,1 \cdot 88 \\
1 \cdot 26,2 \cdot 00 \\
0.36,0.55 \\
0.26,0.46\end{array}$ & & \\
\hline $\begin{array}{l}\text { Model 2: residence } \times \text { gender } \\
\text { Gender (ref.: women) }\end{array}$ & & & & & 0.131 & 0.085 \\
\hline $\begin{array}{l}\text { Men } \\
\text { Residence (ref.: rural) }\end{array}$ & $1 \cdot 11$ & $0.96,1.27$ & $0.37^{\star \star \star}$ & $0.30,0.46$ & & \\
\hline $\begin{array}{l}\text { Urban } \\
\text { (Men } v \text {. women) } \times \text { urban }\end{array}$ & $\begin{array}{l}0.73^{\star \star \star} \\
1.33^{\star \star \star}\end{array}$ & $\begin{array}{l}0.64,0.84 \\
1.07,1.64\end{array}$ & $\begin{array}{l}1.55^{\star \star *} \\
0.46^{\star \star \star}\end{array}$ & $\begin{array}{l}1.34,1.78 \\
0.37,0.57\end{array}$ & & \\
\hline $\begin{array}{l}\text { Model 3: education } \times \text { gender } \\
\text { Gender (ref.: women) }\end{array}$ & & & & & 0.165 & $0.004^{\star * *}$ \\
\hline $\begin{array}{l}\text { Men } \\
\text { Education (ref.: no education) }\end{array}$ & $1 \cdot 27^{\star \star \star}$ & $1.07,1.51$ & $0 \cdot 25^{\star \star \star}$ & $0.17,0.27$ & & \\
\hline $\begin{array}{l}\text { Primary } \\
\text { Secondary or higher } \\
\text { (Men } v \text {. women) } \times \text { primary } \\
\text { (Men } v \text {. women) } \times \text { secondary or higher }\end{array}$ & $\begin{array}{l}0 \cdot 88^{\star \star \star} \\
0 \cdot 75^{\star \star \star} \\
1.07 \\
1.04\end{array}$ & $\begin{array}{l}0.78,0.99 \\
0.66,0.85 \\
0.89,1.28 \\
0.85,1.28\end{array}$ & $\begin{array}{l}1 \cdot 3^{\star \star \star} \\
1 \cdot 69^{\star \star \star} \\
0.37^{\star \star \star} \\
0.48^{\star \star \star}\end{array}$ & $\begin{array}{l}1.12,1.52 \\
1.48,1.95 \\
0.28,0.49 \\
0.39,0.58\end{array}$ & & \\
\hline $\begin{array}{l}\text { Model 4: wealth } \times \text { gender } \\
\text { Gender (ref,: women) }\end{array}$ & & & & & 0.549 & $0.02^{\star \star \star}$ \\
\hline $\begin{array}{l}\text { Men } \\
\text { Wealth (ref.: poor) }\end{array}$ & 1.09 & $0.93,1.27$ & $0 \cdot 24^{\star \star *}$ & $0.15,0.37$ & & \\
\hline $\begin{array}{l}\text { Middle } \\
\text { Rich } \\
\text { (Men } v \text {. women }) \times \text { middle } \\
\text { (Men } v \text {. women }) \times \text { rich }\end{array}$ & $\begin{array}{l}0 \cdot 73^{\star \star \star} \\
0 \cdot 48^{\star \star \star} \\
1.20 \\
1.20\end{array}$ & $\begin{array}{l}0.64,0.83 \\
0.42,0.54 \\
0.97,1.47 \\
0.99,1.46\end{array}$ & $\begin{array}{l}1 \cdot 50^{\star \star \star} \\
3 \cdot 20^{\star \star \star} \\
0.41^{\star \star *} \\
0.45^{\star \star *}\end{array}$ & $\begin{array}{l}1.23,1.81 \\
2.76,3.71 \\
0.29,0.59 \\
0.37,0.54\end{array}$ & & \\
\hline
\end{tabular}

Ref., reference category.

Model 1 adjusts for residence, education, wealth tertile, marital status, region, working status plus two-way interaction between age and gender. Model 2 adjusts for age, education, wealth tertile, marital status, region, working status plus two-way interaction between residence and gender. Model 3 adjusts for age, residence, wealth tertile, marital status, region, working status plus two-way interactions between education and gender. Model 4 adjusts for age, residence, education, marital status, region, working status plus two-way interaction between wealth tertile and gender. ${ }^{\star \star \star} P<0.001$.

urban residence and higher wealth status played a significantly protective role against increasing BMI for men compared with rural and poor women, respectively.

Women were found to be more overweight and less underweight than men in the present study. However, more educated women who were wealthier and living in rural areas had higher BMI and a higher overweight prevalence than men from corresponding groups. These findings may represent the tendency of women in this context to stay at home and be less physically active. This increased risk may also be due to preferences for carbohydrate- and fat-based foods and the existence of various social and cultural barriers to physical activity among women. Bangladesh has the highest levels of reported physical inactivity and poor dietary habits among South Asian countries ${ }^{(26)}$, although no such study is available to compare the physical activity and dietary habits among men and women.

The present study found large disparities between the highest and lowest tertiles of wealth status for both genders. Thus higher wealth status was a protective factor for underweight but a risk factor for overweight. On the other hand, lower wealth status worked as a protective factor against overweight, a finding which is consistent with other studies ${ }^{(2,18,27-29)}$. The observation that higher educated subgroups had the highest prevalence of overweight and lowest prevalence of underweight, which was reversed for subgroups with no education, is consistent with other studies ${ }^{(8,15)}$. However, urban residence, higher wealth status and higher education increased the risk of women being overweight. The findings for women of higher wealth status may result from their having a more sedentary lifestyle and their preferences for energy-dense foods compared with men in this same group ${ }^{(29)}$.

The study suggests that adults living in urban areas were more likely to be overweight than rural adults. On the other hand, adults living in rural areas were more likely to be underweight than adults living in urban areas. These findings are consistent with similar analyses conducted in Bangladesh $^{(7)}$ and other developing countries ${ }^{(18,30-32)}$. 
Table 4 Adjusted and unadjusted mean BMI $\left(\mathrm{kg} / \mathrm{m}^{2}\right)$ among men and women, Bangladesh Demographic and Health Survey 2011

\begin{tabular}{|c|c|c|c|c|c|c|c|c|}
\hline & \multicolumn{4}{|c|}{ Men } & \multicolumn{4}{|c|}{ Women } \\
\hline & \multicolumn{2}{|c|}{ Unadjusted } & \multicolumn{2}{|c|}{ Adjusted } & \multicolumn{2}{|c|}{ Unadjusted } & \multicolumn{2}{|c|}{ Adjusted } \\
\hline & $\begin{array}{l}\text { Mean or } \\
\text { coefficient }\end{array}$ & $95 \% \mathrm{Cl}$ & $\begin{array}{l}\text { Mean or } \\
\text { coefficient }\end{array}$ & $95 \% \mathrm{Cl}$ & $\begin{array}{l}\text { Mean or } \\
\text { coefficient }\end{array}$ & $95 \% \mathrm{Cl}$ & $\begin{array}{l}\text { Mean or } \\
\text { coefficient }\end{array}$ & $95 \% \mathrm{Cl}$ \\
\hline \multicolumn{9}{|l|}{ Age category } \\
\hline 19-34 years & 20.52 & $20 \cdot 34,20 \cdot 71$ & Ref. & & $21 \cdot 30$ & $21 \cdot 18,21 \cdot 42$ & Ref. & \\
\hline $35-49$ years & $0.45^{\star \star \star}$ & $0.21,0.70$ & $0.50^{\star \star \star}$ & $0.27,0.73$ & $0.52^{\star \star \star}$ & $0.36,0.67$ & $0.81^{\star \star \star}$ & $0.66,0.96$ \\
\hline$\geq 50$ years & $-0.53^{\star \star \star}$ & $-0.76,-0.31$ & $-0 \cdot 13^{*}$ & $-0.37,0.09$ & $-0 \cdot 76^{\star \star \star}$ & $-1.02,-0.51$ & 0.12 & $-0.26,0.29$ \\
\hline \multicolumn{9}{|l|}{ Residence } \\
\hline Rural & 20.09 & $19 \cdot 96,20 \cdot 22$ & Ref. & & $20 \cdot 85$ & $20 \cdot 73,20 \cdot 98$ & Ref. & \\
\hline Urban & $1.60^{\star \star \star}$ & $1.31,1.89$ & $0.54^{\star \star \star}$ & $0.26,0.81$ & $2 \cdot 26^{\star \star \star}$ & $1.98,2.54$ & $1.06^{\star \star \star}$ & $0.82,1.30$ \\
\hline \multicolumn{9}{|l|}{ Wealth tertile } \\
\hline Poor & $19 \cdot 33$ & $19.20,19.46$ & Ref. & & 19.92 & $19 \cdot 82,20 \cdot 03$ & Ref. & \\
\hline Middle & $0.73^{\star \star *}$ & $0.49,0.96$ & $0.49^{\star \star \star}$ & $0.25,0.72$ & $1.02^{\star \star \star}$ & $0.85,1.19$ & $0.73^{\star \star \star}$ & $0.56,0.90$ \\
\hline Rich & $2 \cdot 49^{\star \star \star}$ & $1 \cdot 26,2 \cdot 72$ & $1 \cdot 74^{\star \star \star}$ & $1.48,1.99$ & $3 \cdot 10^{\star \star \star}$ & $2 \cdot 92,3 \cdot 29$ & $2 \cdot 27^{\star \star \star}$ & $2.09,2.45$ \\
\hline \multicolumn{9}{|c|}{ Highest education level } \\
\hline No education & 19.38 & $19.24,19.53$ & Ref. & & 20.54 & $20 \cdot 40,20.67$ & Ref. & \\
\hline Primary & $0.85^{\star \star *}$ & $0.63,1.06$ & $0.49^{\star \star \star}$ & $0.27,0.70$ & $0.65^{\star \star \star}$ & $0.48,0.83$ & $0.36^{\star \star \star}$ & $0.18,0.53$ \\
\hline $\begin{array}{l}\text { Secondary or } \\
\text { higher }\end{array}$ & $2 \cdot 31^{\star \star *}$ & $2.07,2.54$ & $1 \cdot 30^{* * *}$ & $1.05,1.55$ & $1.96^{\star \star *}$ & $1 \cdot 76,2 \cdot 15$ & $0.93^{\star \star \star}$ & $0.74,1.13$ \\
\hline \multicolumn{9}{|l|}{ Marital status } \\
\hline Not married & $19 \cdot 45$ & $18 \cdot 84,20.05$ & Ref. & & $20 \cdot 62$ & $20 \cdot 39,20 \cdot 85$ & Ref. & \\
\hline Currently married & $1.06^{* *}$ & $0.46,1.66$ & $0.74^{\star *}$ & $0.20,1.29$ & $0.91^{* \star \star}$ & $0.67,1.13$ & $0.51^{\star \star \star}$ & $0.29,0.73$ \\
\hline \multicolumn{9}{|l|}{ Working status } \\
\hline Currently working & $19 \cdot 79$ & $19 \cdot 46,20 \cdot 12$ & Ref. & & 21.42 & $21 \cdot 30,21 \cdot 54$ & Ref. & \\
\hline Not working & $0.77^{\star \star \star}$ & $0.45,1.10$ & $0.45^{\star \star}$ & $0.11,0.78$ & 0.02 & $-0.20,0.25$ & -0.14 & $-0.34,0.05$ \\
\hline
\end{tabular}

Ref., reference category.

${ }^{\star} P<0.05,{ }^{\star \star} P<0.01,{ }^{\star \star \star} P<0.001$.

This high prevalence of adult overweight in urban areas could result from recent urbanization ${ }^{(33)}$ and economic growth of the country. Urbanization is associated with a transition of the traditional lifestyle to a more sedentary one with increased reliance on automobiles, consuming ready-to-eat energy-dense foods and decline of physical activity due to lack of parks and walkways. All of these factors may have a possible impact on the higher prevalence of overweight among urban adults.

The men $v$. women interaction with urban/rural residence showed that the odds of overweight were lower and the odds of underweight were higher for urban men compared with urban women. The findings of higher prevalence of overweight among urban women than men may due to the cultural practice of urban women of staying at home more and sedentary lifestyle. Widespread access to television and the Internet would also favour an indoor, sedentary lifestyle for the women. Nationally $42.2 \%$ of households have a television ${ }^{(6)}$ and $14.5 \%$ of people are Internet users ${ }^{(21)}$.

The present study has several important strengths and limitations. The main strength of our study is that we used a large nationally representative sample of an adult population. The cross-sectional nature of the data limits our ability to discuss the causal relationships of the associations among the observed factors, although the study did not attempt to carry out a causal analysis. A number of study limitations are worth to be addressed. First, we used BMI as the measure of adult nutritional status, although BMI fails to differentiate between body fat and lean body mass. Additionally, the amount of body fat differs between men and women ${ }^{(34)}$. However, among the various anthropometric measures, BMI is considered more appropriate to predict underweight- and overweightrelated determinants at population level ${ }^{(35)}$. Finally we did not consider information on dietary data, physical activity and energy intake due to lack of such information in the BDHS.

\section{Conclusion}

In conclusion, the current study highlights the existence of the double burden of both underweight and overweight among Bangladeshi men and women, with underweight more prevalent among the rural, poor and uneducated while overweight is more prevalent among the urban, rich and educated. Women with higher educational attainment, rich and with urban residence were at greater odds of being overweight compared with their male counterparts with similar characteristics. Thus it is necessary to consider interventions that could control and reduce the prevalence of underweight while simultaneously preventing the increase of overweight prevalence among women in Bangladesh. The study also exposes that the overweight adult exists mainly in urban areas whereas the rural adult still has a higher burden of underweight. Extensive national campaigns and operational research are needed 
Table 5 Adjusted mean BMI $\left(\mathrm{kg} / \mathrm{m}^{2}\right)$ in interaction models among men and women, Bangladesh Demographic and Health Survey 2011

\begin{tabular}{|c|c|c|c|}
\hline & $\begin{array}{l}\text { Adjusted } \\
\text { coefficient }\end{array}$ & $95 \% \mathrm{Cl}$ & $\begin{array}{c}\text { Overall test of } \\
\text { interaction } \\
(P \text { value })\end{array}$ \\
\hline \multicolumn{3}{|l|}{$\begin{array}{l}\text { Model 1: age } \times \text { gender } \\
\text { Gender (ref. } \text { women) }\end{array}$} & 0.019 \\
\hline Men & $-0 \cdot 72^{\star \star \star}$ & $-0.96,-0.47$ & \\
\hline \multicolumn{4}{|l|}{ Age category (ref.: 19-34 years) } \\
\hline $35-49$ years & 0.85 & $0.70,1.00$ & \\
\hline$\geq 50$ years & $0.08^{\star \star \star}$ & $-0.20,0.35$ & \\
\hline (Men $v$. women) $\times 35-49$ years & $-1.08^{\star \star \star}$ & $-1.31,-0.86$ & \\
\hline (Men $v$. women) $\times 50$ years and above & $-1.06^{\star \star \star}$ & $-1 \cdot 35,-0.77$ & \\
\hline \multicolumn{3}{|l|}{$\begin{array}{l}\text { Model 2: residence } \times \text { gender } \\
\text { Gender (ref.: women) }\end{array}$} & $<0.001$ \\
\hline Men & $-0 \cdot 74^{\star \star \star}$ & $-0.94,-0.55$ & \\
\hline \multicolumn{4}{|l|}{ Residence (ref.: rural) } \\
\hline Urban & $1 \cdot 10$ & $0.87,1.34$ & \\
\hline (Men $v$. women) $\times$ urban & $-1 \cdot 47^{\star \star \star}$ & $-1 \cdot 73,-1 \cdot 21$ & \\
\hline \multicolumn{3}{|l|}{ Model 3: education $\times$ gender } & 0.924 \\
\hline \multicolumn{4}{|l|}{ Gender (ref.: women) } \\
\hline Men & $-0.97^{\star \star \star}$ & $-1.20,-0.75$ & \\
\hline \multicolumn{4}{|l|}{ Education (ref.: no education) } \\
\hline Primary & $0.38^{\star \star \star}$ & $0.21,0.55$ & \\
\hline Secondary or higher & 1.00 & $0.82,1.18$ & \\
\hline (Men $v$. women) $\times$ primary & $-0.92^{\star \star \star}$ & $-1 \cdot 16,-0 \cdot 69$ & \\
\hline (Men $v$. women) $\times$ secondary or higher & $-0.94^{\star \star \star}$ & $-1.18,-0.69$ & \\
\hline \multicolumn{3}{|l|}{ Model 4 : wealth $\times$ gender } & $<0.001$ \\
\hline \multicolumn{4}{|l|}{ Gender (ref.: women) } \\
\hline Men & $-0.60^{\star \star \star}$ & $-0.82,-0.39$ & \\
\hline \multicolumn{4}{|l|}{ Wealth (ref.: poor) } \\
\hline Middle & $0 \cdot 74^{\star \star \star}$ & $0.57,0.91$ & \\
\hline Rich & $2 \cdot 32^{\star \star \star}$ & $2 \cdot 13,2.49$ & \\
\hline (Men $v$. women) $\times$ middle & $-0.88^{\star \star \star}$ & $-1 \cdot 15,-0 \cdot 61$ & \\
\hline (Men $v$. women) $\times$ rich & $-1 \cdot 29^{\star \star \star}$ & $-1.52,-1.05$ & \\
\hline
\end{tabular}

Ref., reference category.

Model 1 adjusts for residence, education, wealth tertile, marital status, region, working status plus two-way interaction between age and gender. Model 2 adjusts for age, education, wealth tertile, marital status, region, working status plus two-way interaction between residence and gender. Model 3 adjusts for age, residence, wealth tertile, marital status, region, working status plus two-way interactions between education and gender. Model 4 adjusts for age, residence, education, marital status, region, working status plus two-way interaction between wealth tertile and gender. ${ }^{\star * \star} P<0.001$.

to address the epidemic of adult overweight in urban areas and to continue to find new solutions in response to the adult underweight problem in rural areas. As females are more prone to overweight than males, women-friendly cost-effective interventions, emphasizing the significance of diet, food and lifestyle, consistent with the country's socio-economic profile, geographical pattern and cultural values, are the demand of the time.

\section{Acknowledgements}

Acknowledgements: The authors would like to acknowledge MEASURE DHS Data Archive, ICF International for providing access to the BDHS data. Financial support: This research received no specific grant from any funding agency in the public, commercial or not-for-profit sectors. Conflict of interest: None of the authors had a conflict of interest. Authorship: M.E.H. and A.A.M. developed the study concept. M.E.H., A.A.M. and M.T.H. contributed to the study design. All authors participated in the data analysis and discussion. M.E.H. wrote the first draft. K.Z.L., M.T.H., M.R. and A.A.M. critically reviewed the drafts. All authors have seen and approved the final draft of the study. Ethics of buman subject participation: The BDHS obtained informed consent from each participant before starting the interview.

\section{References}

1. Popkin BM (1994) The nutrition transition in low-income countries: an emerging crisis. Nutr Rev 52, 285-298.

2. Khan SH \& Talukder SH (2013) Nutrition transition in Bangladesh: is the country ready for this double burden? Obes Rev 14, Suppl. 2, 126-133.

3. Ahmed T, Hossain M \& Sanin KI (2012) Global burden of maternal and child undernutrition and micronutrient deficiencies. Ann Nutr Metab 61, Suppl. 1, 8-17.

4. Popkin BM \& Gordon-Larsen P (2004) The nutrition transition: worldwide obesity dynamics and their determinants. Int J Obes Relat Metab Disord 28, Suppl. 3, S2-S9.

5. National Institute of Population Research and Training, Mitra and Associates, \& Macro International (1997) Bangladesh Demographic and Health Survey 1996-1997. Dhaka and Calverton, MD: NIPORT, Mitra and Associates, and Macro International.

6. National Institute of Population Research and Training, Mitra and Associates, \& ICF International (2013) Bangladesh 
Demographic and Health Survey 2011. Dhaka and Calverton, MD: NIPORT, Mitra and Associates, and ICF International.

7. Sairenchi T, Iso H, Irie F et al. (2008) Underweight as a predictor of diabetes in older adults: a large cohort study. Diabetes Care 31, 583-584.

8. Zheng W, McLerran DF, Rolland B et al. (2011) Association between body-mass index and risk of death in more than 1 million Asians. $N$ Engl J Med 364, 719-729.

9. Ni Mhurchu C, Rodgers A, Pan WH et al. (2004) Asia Pacific Cohort Studies C: Body mass index and cardiovascular disease in the Asia-Pacific Region: an overview of 33 cohorts involving 310000 participants. Int J Epidemiol 33, 751-758.

10. World Health Organization (2009) Global Health Risks: Mortality and Burden of Disease Attributable to Selected Major Risks. Geneva: WHO.

11. Hoque ME, Long KZ, Niessen LW et al. (2015) Rapid shift toward overweight from double burden of underweight and overweight among Bangladeshi women: a systematic review and pooled analysis. Nutr Rev 73, 438-447.

12. Shafique S, Akhter N, Stallkamp G et al. (2007) Trends of under- and overweight among rural and urban poor women indicate the double burden of malnutrition in Bangladesh. Int J Epidemiol 36, 449-457.

13. Finucane MM, Stevens GA, Cowan MJ et al. (2011) National, regional, and global trends in body-mass index since 1980: systematic analysis of health examination surveys and epidemiological studies with 960 country-years and 9.1 million participants. Lancet 377, 557-567.

14. Ng M, Fleming T, Robinson M et al. (2014) Global, regional, and national prevalence of overweight and obesity in children and adults during 1980-2013: a systematic analysis for the Global Burden of Disease Study 2013. Lancet 384, 766-781.

15. Pierce BL, Kalra T, Argos M et al. (2010) A prospective study of body mass index and mortality in Bangladesh. Int $J$ Epidemiol 39, 1037-1045.

16. Muennig P, Lubetkin E, Jia H et al. (2006) Gender and the burden of disease attributable to obesity. Am J Public Health 96, 1662-1668.

17. Corsi DJ, Kyu HH \& Subramanian SV (2011) Socioeconomic and geographic patterning of under- and overnutrition among women in Bangladesh. J Nutr 141, 631-638.

18. Khan MM \& Kraemer A (2009) Factors associated with being underweight, overweight and obese among ever-married non-pregnant urban women in Bangladesh. Singapore Med J 50, 804-813.

19. Popkin BM, Adair LS \& Ng SW (2012) Global nutrition transition and the pandemic of obesity in developing countries. Nutr Rev 70, 3-21.
20. Bangladesh Statistics Division, Ministry of Planning (2011) Population and Housing Census: Preliminary Results, 2011. Dhaka: BBS.

21. The World Bank (2016) World Bank data, Bangladesh. http://data.worldbank.org/country/bangladesh (accessed July 2016).

22. WHO Expert Consultation (2004) Appropriate body-mass index for Asian populations and its implications for policy and intervention strategies. Lancet 363, 157-163.

23. World Health Organization (1995) Physical Status: The Use and Interpretation of Anthropometry. Report of a WHO Expert Committee. WHO Technical Report Series no. 854. Geneva: WHO.

24. Ertem M, Bahceci M, Tuzcu A et al. (2008) The association between high parity and obesity in women living in southeastern Turkey. Eat Weight Disord 13, e4-e7.

25. Razzaque A, Nahar L, Van Minh H et al. (2009) Social factors and overweight: evidence from nine Asian INDEPTH Network sites. Glob Health Action 2, 1991.

26. Joshi P, Islam S, Pais P et al. (2007) Risk factors for early myocardial infraction in South Asians compared with individuals in other countries. JAMA 297, 286-294.

27. Monteiro CA, Moura EC, Conde WL et al. (2004) Socioeconomic status and obesity in adult populations of developing countries: a review. Bull World Health Organ 82, 940-946.

28. Subramanian SV, Perkins JM, Ozaltin E et al. (2011) Weight of nations: a socioeconomic analysis of women in low- to middle-income countries. Am J Clin Nutr 93, 413-421.

29. Mamun AA \& Finlay JE (2014) Shifting of undernutrition to overnutrition and its determinants among women of reproductive ages in the 36 low to medium income countries. Obes Res Clin Pract 9, 75-86.

30. Garg C, Khan SA, Ansari SH et al. (2010) Prevalence of obesity in Indian women. Obes Rev 11, 105-108.

31. Mendez MA, Monteiro CA \& Popkin BM (2005) Overweight exceeds underweight among women in most developing countries. Am J Clin Nutr 81, 714-721.

32. Subramanian SV \& Smith GD (2006) Patterns, distribution, and determinants of under- and overnutrition: a populationbased study of women in India. Am J Clin Nutr 84, 633-640.

33. Bangladesh Bureau of Statistics, Statistics and Informatics Division (2012) Bangladesh Population and Housing Census 2011. Dhaka: BBS.

34. Anand K, Shah B, Yadav K et al. (2007) Are the urban poor vulnerable to non-communicable diseases? A survey of risk factors for non-communicable diseases in urban slums of Faridabad. Natl Med J India 20, 115-120.

35. Bosy-Westphal A, Geisler C, Onur S et al. (2006) Value of body fat mass vs anthropometric obesity indices in the assessment of metabolic risk factors. Int J Obes (Lond) 30, 475-483. 